\title{
Desarrollo y caracterización de un material compuesto de matriz de aluminio 6061 y partículas de $\mathrm{TiB}_{2}$
}

\author{
P. Egizabal*, M. Merchán*, M. García-de-Cortázar, L.M. Plaza* y A. Torregaray**
}

\begin{abstract}
Resumen
Una de las vías desarrolladas, en los últimos años, para la mejora de las propiedades mecánicas de las aleaciones de aluminio es la de la incorporación de refuerzos cerámicos en forma de partículas cerámicas. El objetivo principal del trabajo ha sido desarrollar el proceso de fabricación de una aleación de aluminio 6061 reforzada con partículas de diboruro de titanio, $\mathrm{TiB}_{2}$, y analizar las propiedades del material obtenido. Se ha observado que el proceso de fabricación planteado, consistente en la fabricación de las partículas por medio del proceso de síntesis autopropagada a alta temperatura SHS (Self propagating high temperature synthesis) y su posterior incorporación a la aleación 6061, es un proceso viable para la fabricación de aleaciones reforzadas adecuadas para la realización de procesos subsiguientes de extrusión o forja. El resultado final es la obtención de un material adecuado para la fabricación de componentes extruidos y que presenta una mejora de sus propiedades de tracción y fatiga, en comparación a la aleación base.
\end{abstract}

\section{Development and characterization of a metal matrix composite of aluminium 6061 and $\mathrm{TiB}_{2}$ particulates}

Abstract

Keywords

\begin{abstract}
The incorporation of ceramic particles has been one of the ways developed to improve the mechanical properties of aluminium alloys during the last years. The main objective of this work has been to develop the manufacturing process of the reinforced aluminium alloy and analyse its mechanical properties and microstructure. Details about an innovative MMC manufacturing process and the results obtained in the development of a new family of $\mathrm{TiB}_{2}$ reinforced aluminium alloys for forging and extrusion applications are presented. It has been observed that the considered manufacturing process, consisting in the fabrication of particles by SHS and their incorporation into the selected alloy, is a feasible process to manufacture reinforced alloys that can be subsequently extruded or forged. The final result is the development of a material suitable for extrusion and forging applications and that presents an improvement of fatigue and tensile properties of the matrix alloy.
\end{abstract}

Metal matrix composites (MMC); Self propagating high temperature synthesis (SHS); Extrusion; Aluminium 6061; $\mathrm{TiB}_{2}$ particles.

\section{INTRODUCCIÓN}

El interés del sector del transporte, en los materiales compuestos de matriz metálica, MCMM, se debe a las posibilidades que ofrecen de sustitución de materiales y diseños con mayor peso y de contribución a la reducción del consumo de combustible mediante el aligeramiento de materiales. El sector aeronáutico está especialmente interesado en el desarrollo de materiales reforzados con partículas debido a su buen balance de propiedades/coste ${ }^{[1]}$.
En los últimos años, gran parte de la investigación en MCMM se ha centrado en el desarrollo de nuevos procesos de fabricación de materiales reforzados con partículas. Ello, se debe a que las mejoras en resistencia mecánica, rigidez, resistencia a la fluencia y desgaste se obtienen con menor coste de fabricación que con otros materiales compuestos reforzados con fibras, whiskers o monofilamentos ${ }^{[2]}$. Las partículas cerámicas más estudiadas han sido óxidos, carburos, nitruros y boruros ${ }^{[3]} \mathrm{y}$, entre estos últimos, el diboruro de titanio, $\mathrm{TiB}_{2}$, es una posibilidad muy

\footnotetext{
* INASMET-Tecnalia, Paseo de Mikeletegi, 2, 20009 San Sebastián. e-mail: pedro.egizabal@inasmet.es

** UPV/EHU, Dpto.Ing. Minera, Met. y Ciencia de Materiales, 48012 Bilbao.
} 
atractiva debido a su estabilidad en el aluminio y su doble acción de afinador de grano y refuerzo[4].

Tradicionalmente, los MCMM han sido producidos por procesos costosos, tales como la pulvimetalurgia, deposición por proyección, aleación mecánica y tecnologías de fundición como la mezcla directa stir casting, compocasting o reocasting. Todas estas tecnologías se basan en la adición de los refuerzos exógenos cerámicos a una matriz de aluminio y presentan inconvenientes relacionados con una pobre mojabilidad por el aluminio y por el elevado coste del proceso ${ }^{[4]}$.

El desarrollo de MCMM compuestos de aleaciones metálicas y partículas creadas in situ ha sido abordado por diferentes grupos de investigación, para mejorar los anteriores inconvenientes y producir materiales con una microestructura homogénea de partículas cerámicas termodinámicamente estables. Algunas de estas técnicas son la oxidación direccional metal/metal (DIMOX) ${ }^{[5]}$, dispersión exotérmica (XD) ${ }^{[6]}$, infiltración por un gas reactivo ${ }^{[7]}$, reacción vapor-líquido-sólido (VLS) ${ }^{[8]}$, síntesis asistida por

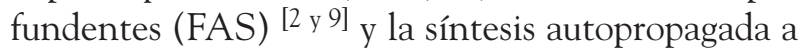
alta temperatura (SHS) ${ }^{[10]}$.

Es conocido que las partículas de $\mathrm{TiB}_{2}$ y de $\mathrm{TiC}$ exhiben ciertas características válidas para el refuerzo de aleaciones de aluminio ${ }^{[11-13]}$. Presentan una gran dureza y resistencia al desgaste y, además, no reaccionan con el metal fundido, evitando la creación de estructuras frágiles como en el caso del carburo de silicio $(\mathrm{SiC})$. La intercara con el aluminio es limpia y exhiben una gran compatibilidad cristalográfica. Además, el elevado valor del coeficiente de expansión térmico (CET) es más cercano al de los valores de las aleaciones de aluminio, disminuyendo la susceptibilidad del material compuesto a la fatiga térmica producido en otros casos (aleaciones de aluminio reforzadas con $\mathrm{SiC}$ o $\mathrm{Al}_{2} \mathrm{O}_{3}$ ), debido a las tensiones residuales generadas en la intercara entre el refuerzo y la matriz de aluminio durante la solidificación.

Diversos autores han producido muestras de $\mathrm{Al}$ $\mathrm{TiC}$ y $\mathrm{Al}-\mathrm{TiB}_{2}$ con hasta un $40 \%$, en volumen, de partículas finas (desde 0,1 hasta 1-2 $\mathrm{mm}$ ) por SHS [14-18]. Sin embargo, estos trabajos están centrados, principalmente, en aspectos relacionados con el proceso, mientras que la influencia del refuerzo en las propiedades mecánicas no ha sido apenas estudiada.

En el presente trabajo se preparó un MCMM por medio de la combinación de la tecnología de SHS y de un proceso convencional de fundición.. El material compuesto fabricado ha sido la aleación de aluminio 6061 reforzada con un $5 \%$, en peso, de partículas de $\mathrm{TiB}_{2}$ que, posteriormente, fue extruído y caracterizado.

\section{MÉTODO EXPERIMENTAL}

\subsection{Fabricación de las partículas por SHS}

Las partículas se fabricaron mediante la tecnología de SHS. Para ello, se mezclaron las cantidades apropiadas de polvo de aluminio, titanio y boro para que mediante SHS se sintetizara el material deseado (aluminio, $30 \% / \mathrm{TiB}_{2}, 70 \%$ ). El aluminio no entra a formar parte de la reacción de síntesis propiamente dicha y se añade, principalmente, para mejorar la mojabilidad de las partículas durante su posterior introducción al aluminio líquido.

\subsection{Fabricación de muestras $6061+\mathrm{TiB}_{2}$}

Para fabricar el material compuesto se utilizó un horno de inducción en el que, primero, se fundió la aleación de aluminio 6061 (Al-1 \% Mg-0,6 \% Si). Posteriormente, se calculó la cantidad de refuerzo obtenido por SHS, necesaria para conseguir un $5 \%$, en peso, de partículas en la mezcla final y se añadió al aluminio líquido, formándose el compuesto 6061$\mathrm{TiB}_{2}$ que fue colado a $730^{\circ} \mathrm{C}$ en moldes cilíndricos de $150 \mathrm{~mm}$ de diámetro y $100 \mathrm{~mm}$ de altura, calentados a $200^{\circ} \mathrm{C}$.

Posteriormente, las muestras cilíndricas de material compuesto se enviaron a la universidad de Bratislava en Eslovaquia (IMMM SAS) para proceder a la etapa de extrusión obteniéndose, finalmente, cilindros de $50 \mathrm{~mm}$ de diámetro y $200 \mathrm{~mm}$ de altura, logrados con una temperatura de extrusión de $450^{\circ} \mathrm{C}$ y una relación de extrusión 9:1 (Fig. 1). Parte del material extraído, fue posteriormente tratado térmicamente por medio de un tratamiento T6 de solubilización a $520^{\circ} \mathrm{C}$ durante $8 \mathrm{~h}$, temple en agua y envejecimiento artificial a $175^{\circ} \mathrm{C}$ durante $8 \mathrm{~h}$.

A partir de los cilindros tratados térmicamente se mecanizaron seis probetas de tracción y otras seis probetas de fatiga.

\subsection{Caracterización}

La caracterización metalúrgica del material compuesto obtenido se realizó mediante microscopía electrónica de barrido (SEM), mientras que para la caracterización mecánica se llevaron a cabo ensayos de tracción según la norma ASTM A370 y de resistencia a fatiga siguiendo la norma ASTM E-466. 


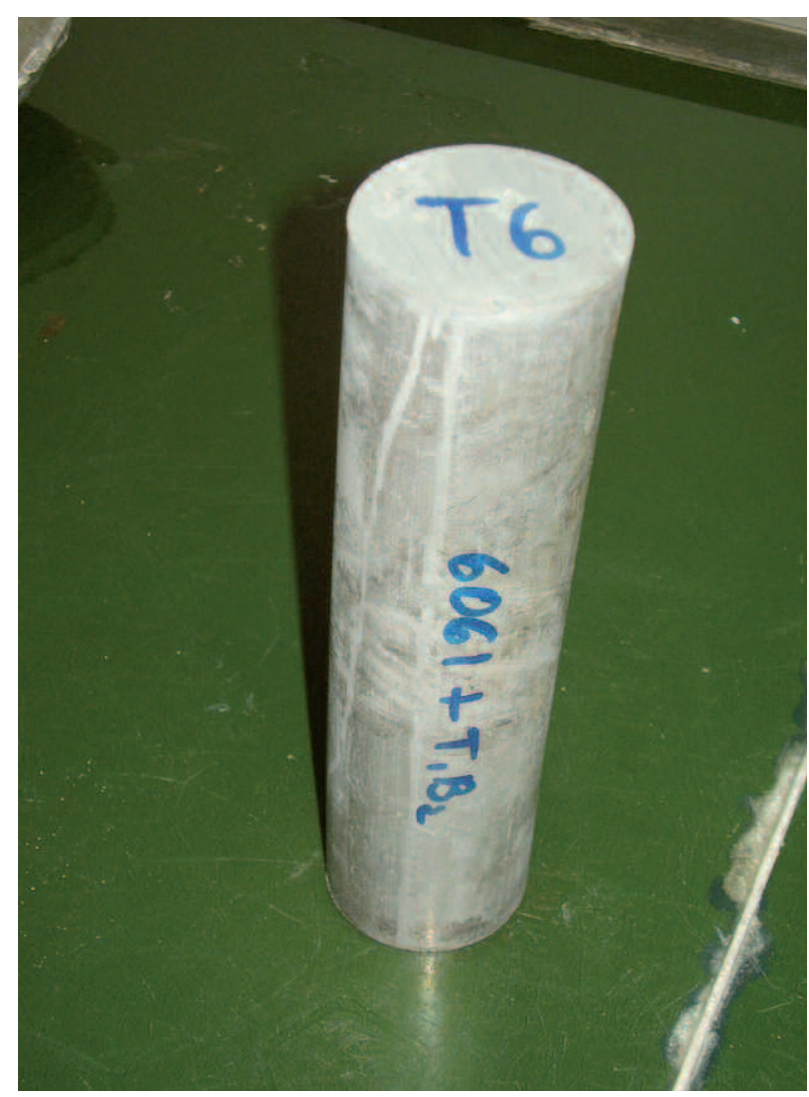

Figura 1. Muestra de aluminio reforzado 6061 $+\mathrm{TiB}_{2}$ extruída y sometida a tratamiento térmico T6.

Figure 1. Sample of the $6061+T i B_{2}$ reinforced alloy extruded and submitted to T6 thermal treatment.

\section{RESULTADOS Y DISCUSIÓN}

En la figura 2, se puede observar una imagen obtenida por microscopía electrónica de barrido en la que se aprecia claramente la distribución de partículas de color gris de $\mathrm{TiB}_{2}$ que aparecen, principalmente, en la frontera de los granos de aluminio junto con otras fases típicas de la aleación 6061. Estas fases son las fases intermetálicas grises de $(\mathrm{Fe}, \mathrm{Mn}) \mathrm{Al}_{6}$ y partículas de $\mathrm{Mg}_{2} \mathrm{Si}$, más oscuras.

El análisis por microscopía óptica confirma la presencia y distribución de estas mismas fases que conforman una microestructura en la que se aprecia la tendencia del $\mathrm{Mg}_{2} \mathrm{Si}$ de formar aglomerados con las partículas de $\mathrm{TiB}_{2}$ (Fig. 3).

En la tabla I se pueden observar los resultados medios obtenidos en los ensayos de tracción realizados tanto a las probetas tratadas térmicamente como a las no tratadas.

Se aprecia claramente la mejora aportada por el tratamiento térmico T6. El límite elástico aumenta

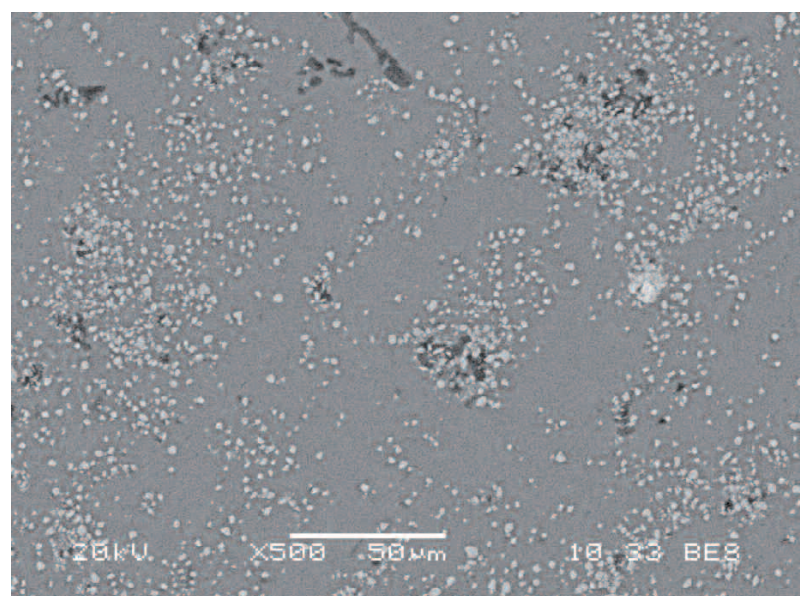

Figura 2. Microestructura obtenida por microscopía electrónica de barrido del material compuesto $6061-5 \% \mathrm{TiB}_{2} \mathrm{~T} 6$ después del proceso de extrusión.

Figure 2. Microstructure obtained by SEM of the $6061+5 \%$ wt. $\mathrm{TiB}_{2}$ reinforced alloy after the extrusion process.

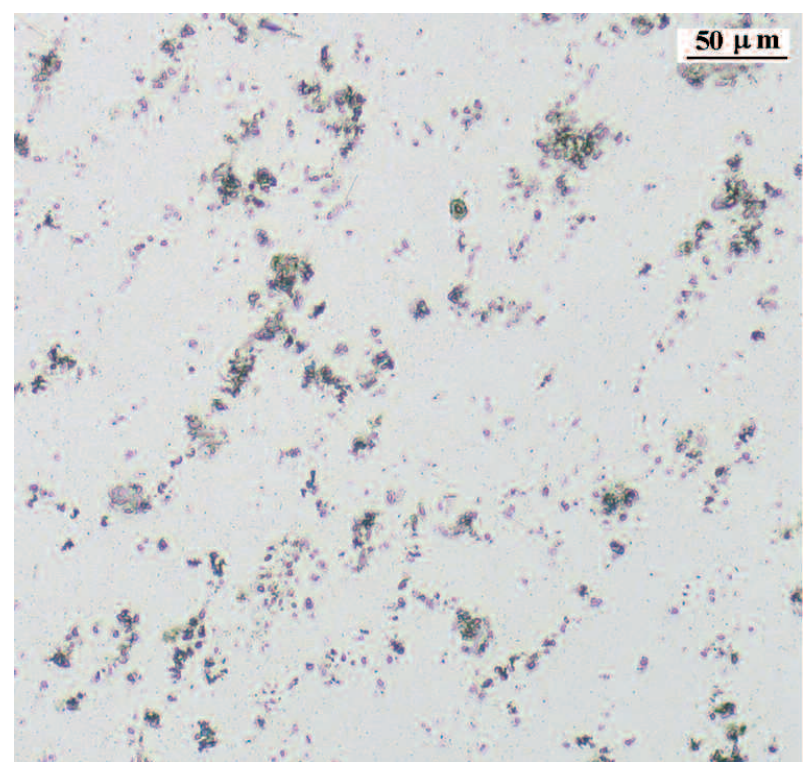

Figura 3. Detalle de la microestructura obtenida por microscopio óptico del material compuesto extruído con presencia de pequeñas aglomeraciones de $\mathrm{TiB}_{2}$ y $\mathrm{Mg}_{2} \mathrm{Si}$.

Figure 3. Detail of the microstructure obtained by optical microscope of the extruded composite material with $\mathrm{Mg}_{2} \mathrm{Si}$ and $\mathrm{TiB}_{2}$ particles forming small agglomerated clusters.

alrededor de un $160 \%$ y la resistencia a tracción aumenta del orden de un $60 \%$. Este incremento de 
DESARROLLO Y CARACTERIZACIÓN DE UN MATERIAL COMPUESTO DE MATRIZ DE ALUMINIO 6061 Y PARTÍCULAS DE TIB 2 DEVELOPMENT AND CHARACTERIZATION OF A METAL MATRIX COMPOSITE OF ALUMINIUM 6061 AND TIB ${ }_{2}$ PARTICULATES

Tabla I. Propiedades mecánicas de $6061+5$ $\% \mathrm{TiB}_{2}$ con su desviación estándar

Table I. Mechanical properties with the corresponding standard deviation of the 6061 $+5 \%$ wt. TiB 2 material

\begin{tabular}{|c|c|c|}
\hline Material & $\begin{array}{c}6061- \\
5 \% \mathrm{TiB}_{2} \text {-T0 }\end{array}$ & $\begin{array}{c}6061- \\
5 \% \mathrm{TiB}_{2}-\mathrm{T} 6\end{array}$ \\
\hline$\sigma_{\mathrm{y}}, \mathrm{MPa}$ & $90,2(3,9)$ & $233,5(6,3)$ \\
\hline$\sigma_{U T S}, \mathrm{MPa}$ & $192,0(2,2)$ & $306,0(1,4)$ \\
\hline Alargamiento, \% & $13,0(0,8)$ & $14,3(1,3)$ \\
\hline
\end{tabular}

propiedades mecánicas es fruto de la homogeneización de la microestructura y de la distribución de las partículas de $\mathrm{TiB}_{2}$ y la precipitación de pequeñas partículas de $\mathrm{Mg}_{2} \mathrm{Si}$ (Figs. 2 y 3 ).

Con las probetas tratadas térmicamente se han realizado ensayos de fatiga a temperatura ambiente, con una relación de cargas de $\mathrm{R}=0,1$, mostrándose los resultados en la tabla II.

Representando los puntos obtenidos en la tabla II en una gráfica S-N, en la que se representan la amplitud de tensiones $\left(\sigma_{\mathrm{a}}\right)$, que es la mitad del intervalo de tensiones $\left(\sigma_{\mathrm{a}}=\left(\sigma_{\max }-\sigma_{\min }\right) / 2\right)$ frente al logaritmo del número de ciclos, se ha obtenido la curva que aparece en la figura 4. Se ha establecido una comparación de la tensión máxima de fatiga entre el material compuesto $6061-\mathrm{TiB}_{2}-\mathrm{T} 6$ y el aluminio comercial 6061-T6, para un determinado número de ciclos, obteniéndose un valor de tensión máxima alrededor de los $175 \mathrm{MPa}$, para una duración de ensayo de 3 millones de ciclos (probeta 5).

Tabla II. Resultados del ensayo de fatiga del material $A 6061+T_{i B}$ según norma ASTM E-466

Table II. Results of the fatigue test corresponding to the $6061+5 \%$ wt. $\mathrm{TiB}_{2}$ ASTM E-466

\begin{tabular}{ccccc}
\hline Probeta & $\begin{array}{c}\sigma_{\max } \\
(\mathbf{M P a})\end{array}$ & $\begin{array}{c}\sigma_{\min } \\
(\mathbf{M P a})\end{array}$ & $\begin{array}{c}\sigma_{\mathbf{a}} \\
(\mathbf{M P a})\end{array}$ & $\mathbf{N}$ \\
\hline 1 & 250 & 25 & 112,5 & $1,05 \mathrm{E}+05$ \\
2 & 200 & 20 & 90 & $3,39 \mathrm{E}+05$ \\
3 & 190 & 19 & 85,5 & $4,55 \mathrm{E}+05$ \\
4 & 185 & 18,5 & 83,2 & $5,61 \mathrm{E}+05$ \\
5 & 175 & 17,5 & 78,75 & $3,00 \mathrm{E}+06$ \\
\hline
\end{tabular}

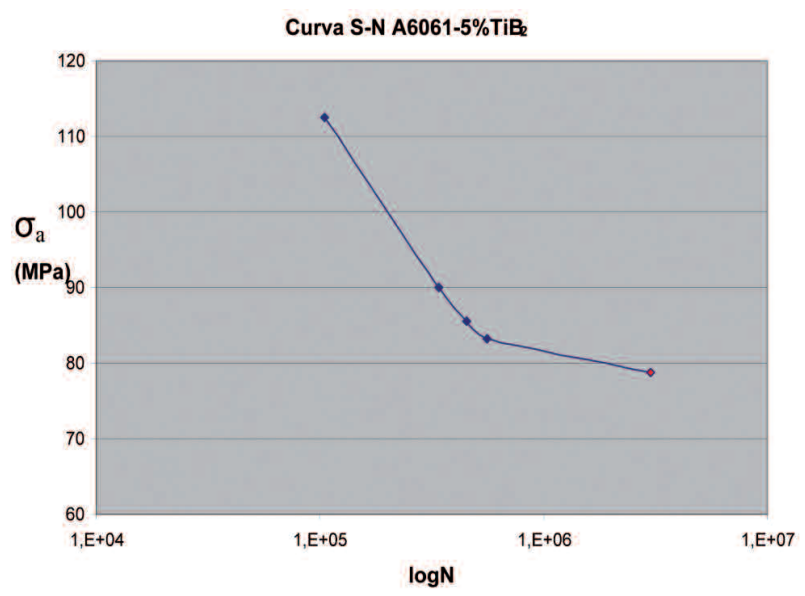

Figura 4. Curva S-N del material compuesto $6061-5 \% \mathrm{TiB}_{2}$ sometido a tratamiento térmico T6.

Figure 4. S-N curve of the reinforced aluminium $6061+5 \%$ wt. TiB 2 submitted to T6 thermal treatment.

En la bibliografía ${ }^{[19]}$ existen datos para una aleación de aluminio 6061 con el tratamiento T6 y una relación de cargas $\mathrm{R}=0$ (Fig. 5). Para una duración de ensayo igual a la de la probeta 5 , la tensión máxima es de $196 \mathrm{MPa}$.

El límite de fatiga del material $6061+5 \%$, en peso, de $\mathrm{TiB}_{2}$ se halla entre 175 y $185 \mathrm{MPa}$ y la resistencia a fatiga del compuesto $\mathrm{A} 6061-\mathrm{TiB}_{2}$, sometido a tratamiento térmico $\mathrm{T} 6$, es de unos $80 \mathrm{MPa}$ al ser

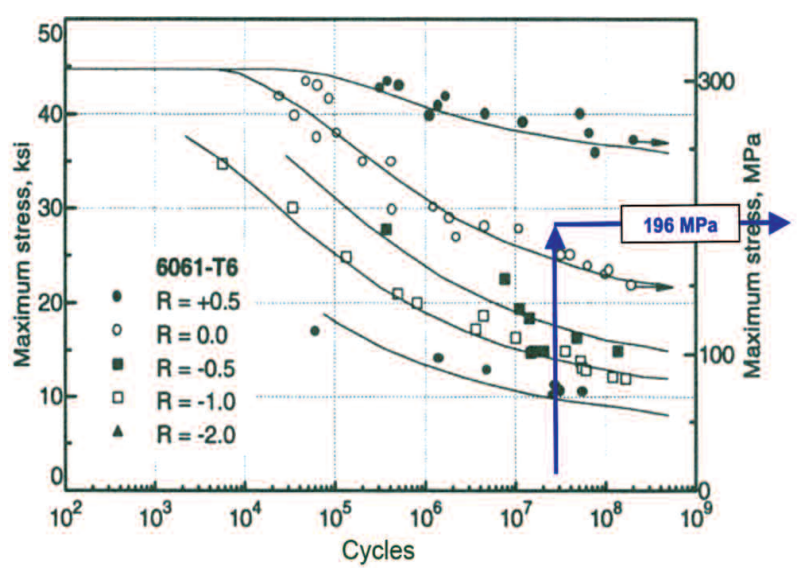

Figura 5. Curvas de una aleación de aluminio 6061 con el tratamiento T6 y una relación de cargas $\mathrm{R}=0{ }^{[19]}$.

Figure 5. Curves corresponding to a 6061 alloy $T 6$ thermally treated and with a load ratio of $R=0$ [19]. 
sometido a un ensayo con un límite de ciclos de $3 \times 10^{6}$ y una relación de cargas de $\mathrm{R}=0,1$.

\section{CONCLUSIONES}

Se ha conseguido fabricar un material compuesto forjable, basado en la aleación 6061 y reforzada con un $5 \%$, en peso, de partículas de $\mathrm{TiB}_{2}$.

El material desarrollado presenta una microestructura con una distribución homogénea de partículas, ausencia de porosidad y defectos en forma de poros o rechupes.

Las muestras del material compuesto A6061$5 \% \mathrm{TiB}_{2}$, tratadas con un tratamiento $\mathrm{T} 6$, presentan una mejora de las propiedades mecánicas con respecto al material sin tratar.

\section{Agradecimientos}

Los autores muestran su agradecimiento al profesor Dr. Simancik del "Institute of Materials \& Machine Mechanics SAS" de la Academia de Ciencias de Eslovaquia por su colaboración en la tarea de puesta a punto de la extrusión del material y fabricación de muestras.

\section{REFERENCIAS}

[1] X.C. Tong, J. Mater. Sci. 33 (1998) 5.365-5.374.

[2] X. Wang, R. Brydson, A. Jha y J. Ellis, J Microsc 196 (1999) 137-145.

[3] K.B. Lee, H.S. Sim y H. Kwon, Metall. Mater. Trans. A 36 (2005) 2.517-2.527.
[4] S.C. Tjong y Z.Y. Ma, Mater. Sci. Eng., A 29 (2000) 49-113.

[5] A.W. Urquhart, Mater. Sci. Eng. A 144 (1991) 75-82.

[6] A.K. Kuruvilla, K.S. Prasad, V.V. Bhanuprasad y Y.R. Mahajan, Scr. Metall. Mater. 24 (1990) 873-878.

[7] P. Sahoo y M.J. Koczak, Mater. Sci. Eng. A 144 (1991) 37-44.

[8] M. J. Koczak y K. S. Kumar, US Patent 4.808.372 (1998).

[9] T. Fan, G. Yang y D. Zhang, Metall. Mater. Trans. A 36 (2005) 225-233.

[10] I. Gotman, M.J. Koczak y E. Shtessel, Mater. Sci. Eng. A 187 (1994) 189-199.

[11] C. Bartels., D. Raabe, G. Gottstein y U. Huber, Mater. Sci. Eng. A 237 (1997) 12-23.

[12] R.M. Aikin, JOM. 49 (1997) 35-39.

[13] M.D. Salvador, V. Amigo, N. Martínez y C. Gerrer, J. Mater. Process. Tech. 143-144 (2003) 598-564.

[14] E. Fras, S. Wierzbinski, A. Janas y H.F. Lopez, Metall. Mater. Trans. A 33 (2002) 3.831-3.838.

[15] M.S. Song, M.X. Zhang, S.G. Zhang, B. Huang y J.G. Li, Mater. Sci. Eng. A 473 (2008) 166171.

[16] P. Li, E.G. Kandalova y V.I. Nikitin, Mater. Lett. 59 (2005) 2.545-2.548.

[17] P. Li, E.G. Kandalova, V.I. Nikitin, A.G. Makarenko, A.R. Luts, Z. Yanfei, Scr. Mater., 49 (2003) 699-703.

[18] K.T. Lee, L. Lu y M.O. Lai, Mater. Sci. Technol. 17 (2001) 201-206.

[19] S.D. Henry, G.M. Davidson, S.R. Lampman, F. Reidenbach, R.L. Boring y W.W. Scott, Fatigue Data Book: Light Structural Alloys, $1^{\underline{a}}$ edición, ASM International, Materials Park, (OH) EE. UU., 1995, pp. 1-397. 